Sánchez Fuentes, S., Martín Almaraz, R.A., Moreno Medina, I. \& Espada Chavarría, R. (2018). Revisión sobre la intervención precoz en dificultades de aprendizaje relacionadas con la lectura. Revista Electrónica Interuniversitaria de Formación del Profesorado, 21(3), 35-45.

\title{
Revisión sobre la intervención precoz en dificultades de aprendizaje relacionadas con la lectura
}

\author{
${ }^{1}$ Sergio Sánchez Fuentes, ${ }^{2}$ Rosario A. Martín Almaraz, ${ }^{1}$ Irene Moreno Medina, ${ }^{2}$ Rosa Espada \\ Chavarría \\ 'Universidad Autónoma de Madrid \\ ${ }^{2}$ Universidad Rey Juan Carlos
}

\section{Resumen}

Este trabajo tiene como objetivo principal realizar una revisión sistemática relacionada con la intervención precoz en la lectura. Para ello, se han analizado las publicaciones desde el año 2000 hasta el 2016 de las bases de datos de la Web of Science, Medline, SCIELO, Korean Journal Database y Current Contents con los términos de búsqueda de Learning disabilities AND Early reading AND Intervention AND Children. De los 113 documentos identificados, finalmente solo 11 son tenidos en cuenta debido a diferentes criterios de exclusión. Los resultados describen que tres artículos son de revisiones y meta-análisis, tres de estudios de cohortes, cuatro de estudios longitudinales y un estudio de casos. Los autores concluyen que existe una necesidad de cambio en el sistema educativo que contemple las medidas destinadas a ayudar a adquirir los mecanismos de base de la lectura en las etapas iniciales del aprendizaje.

\section{Palabras clave}

Lectura; Dislexia; Dificultades de aprendizaje; Intervención.

\section{Contacto:}

Sergio Sánchez Fuentes, sergio.sanchezfuentes@uam.es, Campus Cantoblanco. Facultad de Formación del profesorado. 28049. Madrid 


\title{
Review of early intervention in learning difficulties related to reading
}

\begin{abstract}
The main aim of this paper is to carry out a systematic review related to early intervention in reading. The papers from 2000 to 2016 of the Web of Science, Medline, SCIELO, Korean Journal Database and Current Contents databases have been analyzed by terms of Learning disabilities AND Early reading AND Intervention AND Children. Finally, of the 113 documents identified, only 11 were taken into account due to different exclusion criteria. The results describe that three articles are from reviews and meta-analyzes, three from cohort studies, four from longitudinal studies and one case study. The authors conclude that there is a need for change in the education system that contemplates measures designed to help acquire the basic mechanisms of reading in the initial stages of learning.
\end{abstract}

\section{Key words}

Reading; Dyslexia; Learning disabilities; Intervention

\section{Introducción}

En los primeros años de alfabetización escolar no todos los alumnos siguen el mismo ritmo y algunos estudiantes pueden presentar problemas que si se prolongan en el tiempo pueden tener consecuencias graves. Además, de las consecuencias académicas, varios estudios han investigado acerca de la repercusión de las dificultades de aprendizaje lectoras en la autoestima, la conducta y la adaptación social (Caemmerer y Keith, 2015; Terras, Thompson, y Minnis, 2009; Yu, Buka, McCormick, Fitzmaurice, y Indurkhya, 2006). La importancia de un desarrollo adecuado de la lectura, que pueda procurar lectores eficaces, de forma rápida y económica en cuanto a tiempo y recursos, es crucial.

La detección temprana y la intervención ajustada puede evitar las consecuencias indeseables de repetidas experiencias de fracaso en los estudiantes (Vellutino, Scanlon, Zhang, y Schatschneider, 2008). En nuestro sistema educativo establecido por la LOE (con las modificaciones introducidas por la LOMCE) aparece formulado el principio fundamental de la calidad de la educación para todo el alumnado, en condiciones de equidad y con garantía de igualdad de oportunidades. En las últimas dos décadas la investigación de las dificultades de aprendizaje ha sido de gran interés, y en especial la dirigida al estudio de la dislexia, que además de ayudar a la comprensión de estas dificultades, proporcionan a los educadores información práctica sobre cómo identificar a los niños con necesidades en lectura y la forma de garantizar experiencias exitosas para sus alumnos (Shaywitz, Morris, y Shaywitz, 2008). Según el manual de diagnóstico de referencia mundial publicado por la American Psychiatric Association en su quinta edición, (DSM-V, 2013), el trastorno específico del aprendizaje, se da entre un $5 \%$ y $15 \%$ en los niños de edad escolar. Dentro del trastorno específico del aprendizaje, se encuentran los problemas para leer palabras, los problemas de comprensión lectora, los problemas de pronunciación, los problemas de expresión escrita, los problemas de cálculo y de razonamiento matemático.

Las dificultades académicas pueden generar dificultades en el área social, personal y conductual del estudiante con DEA (Soriano, 1999). En general, la dislexia es una dificultad específica para la lectura producida por alguna disfunción cerebral, y sin razón aparente, es 
decir, se descartan causas como baja inteligencia, ambiente socioeconómico desfavorable, mala escolaridad, enfermedad física (problemas de audición o visión) o psíquica (problemas emocionales graves) que afectan al rendimiento general (Cuetos, 2008). La dislexia se manifiesta de distintas formas en los distintos idiomas, las variaciones que muestra la dislexia en las distintas partes del mundo dependen de factores ambientales $y$, en particular, del sistema de escritura que utiliza (Miles, 2004). Serrano (2005) observa diferencias en el desarrollo y en los problemas de la dislexia evolutiva es castellano respecto a la dislexia en niños ingleses. Así, en los momentos iniciales del aprendizaje los niños disléxicos se benefician de la transparencia que supone el castellano para leer palabras, posteriormente en el período de consolidación el déficit se presenta tanto en precisión como en la velocidad de la lectura. Según Cuetos (2008), se deben trabajar conjuntamente las claves sintácticas y léxicas al tiempo que se lee y se enseña a predecir.

Finalmente, el propósito de este trabajo es revisar las investigaciones actuales referidas a la intervención en lectura en las primeras edades de aquellos estudiantes que tienen dificultades en la adquisición de la lectura sin una causa aparente que lo justifique, y extraer las tendencias en la investigación sobre las orientaciones prácticas más beneficiosas y eficaces en el aula con el fin de orientar las actuaciones docentes dirigidas a evitar el fallo en primaria y, en general, aumentar el rendimiento académico de todos los estudiantes, tengan o no dificultades.

\section{Método}

Se realizó una revisión sistemática de las publicaciones científicas que abordan el tema de la intervención temprana en dificultades de aprendizaje en lectura. Para la búsqueda de artículos científicos se consultaron las bases de datos de Web of Science, Medline, SCIELO, Korean Journal Database y Current Contents, delimitando la búsqueda al período de publicación 2000 hasta 2016 y en el idioma inglésEn la búsqueda se utilizó Learning disabilities AND Early reading AND Intervention AND Children.

\section{Participantes}

En la selección de los artículos se estableció como criterio de inclusión básico el hecho de que se tomara como objeto de estudio la intervención en dificultades de aprendizaje de lectura de forma temprana ( 3 a 8 años).

\section{Procedimiento}

Para proceder a la selección se revisaron los abstracts y en caso necesario los artículos completos con el fin de decidir si la información que contenían estaba o no relacionada con el objetivo planteado. La información seleccionada se estructuró en dos subapartados: uno dedicado al diagnóstico, detección e identificación (intervención preventiva), y otro dedicado a la intervención propiamente dicha, y objeto de este trabajo de revisión. Del conjunto de estudios que tratan el tema de la intervención, se extrajo información para su análisis de las siguientes variables: autoría, año y país de la publicación, tipo de estudio, muestra analizada (edad, nivel/grado de estudios y origen), medida de resultado y conclusiones.

\section{Resultados}

Con esta estrategia de búsqueda se identificaron 115 documentos de los cuales 105 correspondieron a la base Web of Science, cinco artículos en la base Medline, tres en 
Korean Journal Datanase, uno en SCIELO y uno en Current Contents. En los documentos inicialmente identificados se localizaron dos duplicados, siendo el total de la muestra 113 artículos. Tras una revisión inicial se excluyeron 79 estudios que no fueron relevantes para el objetivo de esta revisión Siguiendo los criterios de selección y exclusión, los estudios excluidos han sido considerados como no significativos para el objeto de esta revisión, por los siguientes motivos: i. Estudios en sistemas de escritura no alfabética (7); ii. Tema incongruente (7); iii: Artículos teóricos o genéricos (17); iv: Referidos a enfermedades (9); v: Factores genéticos (5); vi: Factores contextuales (4); vii: Discapacidad (11); viii: Refereidos al lenguaje oral (10); y ix: Habilidades sociales (9).

El total de artículos seleccionados se agruparon en las categorías de diagnóstico y/o detección y la categoría de intervención, con 12 y 22 estudios respectivamente.

De ellos se extrajeron las variables año de publicación, tipo de estudio, país de publicación (Tabla 1). Entre los años 2008 y 2011 se encuentra la mayoría de las publicaciones. Los estudios se refieren, preferentemente, a investigaciones descriptivas con aplicación de análisis cuantitativos de los datos. En cuanto a los países de publicación la mayoría de los estudios publicados en inglés son de EEUU, otros países de publicación son España e Israel con dos estudios cada uno, y Alemania, Australia y Brasil con uno cada uno.

Tabla 1.

Variables año de publicación, tipo de estudio y país. (Elaboración propia)

\begin{tabular}{|c|c|c|c|c|}
\hline Variables & & $\begin{array}{l}\text { Detección } \\
\text { n } 1=12\end{array}$ & $\begin{array}{l}\text { Intervención } \\
\text { n } 2=\mathbf{2 2}\end{array}$ & $\begin{array}{l}\text { Total } \\
\mathrm{n} 1+\mathrm{n} 2\end{array}$ \\
\hline \multirow{8}{*}{$\begin{array}{l}\text { Año } \\
\text { publicación }\end{array}$} & 2000 y 2001 & 0 & 2 & 2 \\
\hline & 2002 y 2003 & 1 & 3 & 4 \\
\hline & 2004 y 2005 & 0 & 1 & 1 \\
\hline & 2006 y 2007 & 2 & 1 & 3 \\
\hline & 2008 y 2009 & 3 & 4 & 7 \\
\hline & 2010 y 2011 & 4 & 7 & 11 \\
\hline & 2012 y 2013 & 1 & 1 & 2 \\
\hline & 2014 y 2015 & 1 & 3 & 4 \\
\hline \multirow{6}{*}{$\begin{array}{l}\text { Tipo de } \\
\text { estudio }\end{array}$} & Cualitativos & & 6 & \\
\hline & Cuantitativos & & 16 & \\
\hline & - Review & & 6 & \\
\hline & - Cohortes & & 4 & \\
\hline & - Longitudinal & & 4 & \\
\hline & - Casos & & 2 & \\
\hline \multirow[t]{6}{*}{ Países } & EEUU & 10 & 17 & 27 \\
\hline & España & - & 2 & 2 \\
\hline & Israel & 1 & 1 & 2 \\
\hline & Alemania & - & 1 & 1 \\
\hline & Australia & - & 1 & 1 \\
\hline & Brasil & 1 & - & 1 \\
\hline
\end{tabular}


Para facilitar el posterior análisis de las variables de los estudios de intervención temprana inicialmente seleccionados se agrupan en tres bloques, en función del tipo de estudio: el primero compuesto por seis estudios de revisiones y meta-análisis; un segundo bloque referido a los estudios de cohortes, de casos y descriptivos longitudinales; y un tercer bloque a los estudios cualitativos. En cada uno de ellos se analizan las siguientes variables: en las revisiones y meta-análisis las variables finalidad, muestra y conclusiones; en el bloque referido a los estudios de cohortes, de casos y descriptivos longitudinales las variables país, tipo de estudio, muestra, resultados y conclusiones; y en los estudios cualitativos se extraen las ideas principales.

El análisis de las variables lleva a excluir cinco estudios. Tres del primer bloque denominado revisiones y meta-análisis, los motivos son: se refiere a detección de dificultades (Carreker, 2010), trata la relación lectura y habilidades sociales (Roberts, 2015) y aborda si las diferencias iniciales del alumnado en lectura se mitigan con la intervención (Tran, 2011); dos artículos más se excluyen por no tratar aspectos preventivos y referirse a tratamientos en Educación Especial (Jitendra, 2004; O'Connor 2002). También se excluyen los estudios cualitativos.

Finalmente se seleccionan aquellos estudios empíricos cuantitativos cuyo tema es la intervención temprana en el ámbito escolar ordinario en un sistema alfabético, dando un total de 11 artículos: tres de revisiones y meta-análisis, tres estudios de cohortes, cuatro estudios longitudinales y un estudio de casos. En las siguientes tablas se pueden consultar de manera más detalla los resultados encontrados.

Tabla 2.

Variables año de publicación, tipo de estudio y país. (Elaboración propia)

\begin{tabular}{|c|c|c|c|}
\hline Autor, año, fuente & Finalidad & Muestra & Conclusiones \\
\hline $\begin{array}{l}\text { Suggate, } \\
2010 \\
\text { Developmental } \\
\text { Psychology }\end{array}$ & $\begin{array}{l}\text { Tamaño del efecto } \\
\text { de la intervención, } \\
\text { moderado por la } \\
\text { modalidad de la } \\
\text { intervención y } \\
\text { desarrollo de } \\
\text { lectores } \\
\text { desfavorecidos. }\end{array}$ & $\begin{array}{l}85 \text { estudios } \\
\text { experimentales o } \\
\text { cuasiexperimentales } \\
\text { estudiante de } \\
\text { preescolar hasta grado } \\
7(\mathrm{~K}-\mathrm{7})\end{array}$ & $\begin{array}{l}\text { Tamaños del efecto mayores } \\
\text { para los estudiantes de } \\
\text { mayor edad y las } \\
\text { intervenciones de } \\
\text { comprensión, en estudios } \\
\text { cuasiexperimentales y } \\
\text { muestras en mayor riesgo. } \\
\text { Intervenciones fonéticas } \\
\text { fueron más efectivas hasta } \\
\text { grado 1, después en } \\
\text { intervenciones en } \\
\text { comprensión, y mixtas, en } \\
\text { particular. }\end{array}$ \\
\hline $\begin{array}{l}\text { Wanzek y Vaughn, } \\
2007 \\
\text { School } \\
\text { Psychology } \\
\text { Review }\end{array}$ & $\begin{array}{l}\text { Síntesis } \\
\text { investigaciones en } \\
\text { intervenciones } \\
\text { tempranas de } \\
\text { lectura para los } \\
\text { estudiantes con } \\
\text { dificultades de } \\
\text { lectura y } \\
\text { discapacidad. }\end{array}$ & $\begin{array}{l}18 \text { estudios publicados } \\
\text { entre } 1995 \text { y } 2005\end{array}$ & $\begin{array}{l}\text { Resultados positivos para los } \\
\text { estudiantes que participan en } \\
\text { las intervenciones extensas, } \\
\text { efectos más altos para la } \\
\text { intervención en el grupo más } \\
\text { pequeño, así como para la } \\
\text { intervención temprana (K-1) }\end{array}$ \\
\hline $\begin{array}{l}\text { Weiser y Mathes, } \\
2011 \\
\text { Review of } \\
\text { Educational }\end{array}$ & $\begin{array}{l}\text { Dar apoyo empírico } \\
\text { a la instrucción de } \\
\text { codificación directa, } \\
\text { explícita para }\end{array}$ & $\begin{array}{l}\mathrm{N}=11 \text { estudios } \\
\text { experimentales o de } \\
\text { control de alta calidad }\end{array}$ & $\begin{array}{l}\text { Evidencia empírica para } \\
\text { apoyar el uso de la } \\
\text { instrucción de codificación; } \\
\text { puede aumentar el }\end{array}$ \\
\hline
\end{tabular}




\begin{tabular}{lll}
\hline Research & aumentar las & rendimiento de los \\
capacidades de L-E & estudiantes en riesgo (grado \\
de los estudiantes & 1, primaria) y mejorar las \\
de primaria en & actuaciones de L y E de los \\
riesgo de fracaso de & estudiantes mayores con \\
alfabetización & problemas de aprendizaje. \\
\hline
\end{tabular}

Tabla 3.

Resultados de estudios empíricos cuantitativos de intervención temprana: cohortes

\begin{tabular}{|c|c|c|c|}
\hline $\begin{array}{l}\text { Autor, año, } \\
\text { país }\end{array}$ & Muestra & Propósito & Conclusiones \\
\hline $\begin{array}{l}\text { Mioduser, } \\
2000 \\
\text { EEUU } \\
\text { Cohorte }\end{array}$ & $\begin{array}{l}\mathrm{N}=46 \text { niños ( } 5- \\
6 \text { años) en alto } \\
\text { riesgo, en tres } \\
\text { grupos de } \\
\text { tratamiento }\end{array}$ & $\begin{array}{l}\text { Contribución de } \\
\text { instrucción basada en } \\
\text { computadoras a la } \\
\text { adquisición de las } \\
\text { habilidades de lectura } \\
\text { temprana, así como los } \\
\text { efectos de las } \\
\text { características } \\
\text { específicas de la } \\
\text { tecnología informática } \\
\text { en el rendimiento } \\
\text { temprana habilidades de } \\
\text { lectura. }\end{array}$ & $\begin{array}{l}\text { Los niños con alto riesgo que } \\
\text { recibieron el programa de } \\
\text { intervención de lectura con } \\
\text { materiales informáticos } \\
\text { mejoraron significativamente } \\
\text { su conciencia fonológica, } \\
\text { reconocimiento de palabras y } \\
\text { habilidades nombrar letras } \\
\text { relación con sus pares que } \\
\text { recibieron otro programa de } \\
\text { intervención de lectura. }\end{array}$ \\
\hline $\begin{array}{l}\text { O'Connor, } \\
2014 \\
\text { EEUU } \\
\text { Cohorte }\end{array}$ & $\begin{array}{l}\mathrm{N}=214, \mathrm{~K}-2^{\circ} \\
\mathrm{Nc}=208,1^{\circ} \text { y } 2^{\circ} \\
\mathrm{N} \text { control }=102 \\
2^{\circ}\end{array}$ & $\begin{array}{l}\text { Resultados de acceso a } \\
\text { modelo RTI en la } \\
\text { guardería o en primer } \\
\text { grado en el rendimiento } \\
\text { de lectura de fin de } \\
\text { Grado2 y la colocación } \\
\text { en EE. }\end{array}$ & $\begin{array}{l}\text { Resultados significativamente } \\
\text { más altos en el logro de } \\
\text { lectura al final de grado }\end{array}$ \\
\hline $\begin{array}{l}\text { Vellutino, } \\
2008 \\
\text { EEUU } \\
\text { Cohortes } \\
\text { Longitudinal }\end{array}$ & $\begin{array}{l}\mathrm{N}=1373 \\
\text { Infantiles, clase } \\
\text { media, } \\
\mathrm{n}=462 \\
\text { identificados } \\
\text { inicialmente en } \\
\text { riesgo de } \\
\text { dificultades de } \\
\text { lectura } \\
\text { temprana (33\% } \\
\text { del total), } \\
\text { muestra } \\
\text { comparación n } \\
=54, \text { de } 1^{\circ} \\
\text { grado } \\
\text { como lectores } \\
\text { de desarrollo } \\
\text { normal }\end{array}$ & $\begin{array}{l}\text { Nivel } 1 \text {, se evaluaron al } \\
\text { inicio del } 1^{\circ} \text { grado, } \\
\text { dicotomizadas en } \\
\text { grupos 'continuo } \\
\text { riesgo" y "que ya no en } \\
\text { riesgo "utilizando una } \\
\text { medida compuesta de } \\
\text { habilidades de nivel } \\
\text { básico de palabras. } \\
\text { Nivel } 2 \text { proyectos } \\
\text { (unoauno clases } \\
\text { particulares de } 30 \\
\text { minutos al día) o } \\
\text { intervención basada en } \\
\text { la escuela durante } 1^{\circ} \\
\text { grado. }\end{array}$ & $\begin{array}{l}\text { Los resultados indicaron que } \\
\text { las medidas de RTI distinguen } \\
\text { de manera más eficaz y con } \\
\text { mayor coherencia entre estos } \\
\text { dos grupos que las medidas } \\
\text { psicométricas. }\end{array}$ \\
\hline
\end{tabular}


Tabla 4.

Resultados de estudios empíricos cuantitativos de intervención temprana: longitudinal

\begin{tabular}{|c|c|c|c|}
\hline $\begin{array}{l}\text { Autor, año, } \\
\text { país, estudio }\end{array}$ & Muestra & Resultados & Conclusiones \\
\hline $\begin{array}{l}\text { Berninger, } \\
2002 \\
\text { EEUU } \\
\text { Longitudinal }\end{array}$ & $\begin{array}{l}1^{\circ} \text { grado } \\
\text { Velocidad de } \\
\text { respuesta }\end{array}$ & $\begin{array}{l}\text { Naturaleza y duración de la } \\
\text { intervención temprana de } \\
\text { intensidad relativamente baja. }\end{array}$ & $\begin{array}{l}\text { Los diferentes perfiles } \\
\text { lingüísticos pueden servir } \\
\text { como posibles señales de } \\
\text { instrucción para mejorar la } \\
\text { lectura de los } \\
\text { respondedores lentos. }\end{array}$ \\
\hline $\begin{array}{l}\text { González } \\
\text { Valenzuela, } \\
2011 \\
\text { España } \\
\text { Longitudinal }\end{array}$ & $\begin{array}{l}\mathrm{N}=56, \mathrm{~K}-2^{\circ}, \text { nivel } \\
\text { sociocultural } \\
\text { medio, } \\
\text { inteligencia } \\
\text { media, en riesgo } \\
\text { de problemas de } \\
\text { aprendizaje. } \\
4 \text { fases evaluación } \\
\text { y } 3 \text { intervención } \\
\text { dos variables ( } \mathrm{R}, \\
\text { W) y dos grupos } \\
\text { (IG, NIG). }\end{array}$ & $\begin{array}{l}\text { Efectos de la aplicación de un } \\
\text { programa de intervención } \\
\text { psicoeducativa de lectura y } \\
\text { escritura en edades tempranas } \\
\text { en niños con riesgo de } \\
\text { problemas de aprendizaje, } \\
\text { hincapié en el desarrollo } \\
\text { psicolingüístico y prioriza las } \\
\text { actividades de lectura y escritura } \\
\text { en el programa escolar. }\end{array}$ & $\begin{array}{l}\text { Mejores puntuaciones en } \\
\text { lectura y escritura y avance } \\
\text { significativamente mayor en } \\
\text { el GI sugieren eficacia a } \\
\text { largo plazo de la } \\
\text { intervención temprana, } \\
\text { sistemática y planificada de } \\
\text { la lengua escrita a través de } \\
\text { componentes } \\
\text { psicolingüísticos. }\end{array}$ \\
\hline $\begin{array}{l}\text { Jiménez, } \\
2010 \\
\text { España } \\
\text { Longitudinal }\end{array}$ & $\begin{array}{l}1.123 \text { niños en } \\
\text { riesgo, de } 1^{\circ}\end{array}$ & $\begin{array}{l}\text { Eficacia Nivel 2, proyectos en } \\
\text { grupos pequeños, } 30 \text { minutos al } \\
\text { día, plan de estudios de } \\
\text { Prevención de DEA (PREDEA) }\end{array}$ & $\begin{array}{l}\text { Puntuaciones más altas en } \\
\text { Early Grade Reading } \\
\text { Assessment (EGRA) en el } \\
\text { tipo de sonido inicial, la } \\
\text { comprensión oral, el } \\
\text { conocimiento sonido de la } \\
\text { letra y la fluidez en la lectura } \\
\text { oral. }\end{array}$ \\
\hline $\begin{array}{l}\text { Kamps, } \\
2008 \\
\text { EEUU } \\
\text { Longitudinal }\end{array}$ & $\begin{array}{l}\mathrm{N}=83 \text { de alto } \\
\text { riesgo de fracaso } \\
\text { en la lectura. }\end{array}$ & $\begin{array}{l}\text { Implementación de la } \\
\text { instrucción de lectura en grupos } \\
\text { pequeños ( } 2^{\circ} \text { y } 3^{\circ} \text { nivel), sesiones } \\
30-40^{\prime} \text { mínimo } 3 \text { veces/semana } \\
\text { durante } 2 \text { años. }\end{array}$ & $\begin{array}{l}\text { Mayor rendimiento en } \\
\text { intervención más explícitas y } \\
\text { dirigidas. }\end{array}$ \\
\hline
\end{tabular}

Tabla 5.

Resultados de estudios empíricos cuantitativos de intervención temprana: casos

\begin{tabular}{lll}
\hline Autor, año, Muestra & Objetivo & \\
País & & \\
\hline
\end{tabular}




\begin{tabular}{|c|c|c|c|}
\hline $\begin{array}{l}\text { Berninger } \\
2000 \\
\text { EEUU }\end{array}$ & $\begin{array}{l}7 \text { niños de } \\
3^{\circ} \text {, de los } \\
\text { que se } \\
\text { obtuvieron } \\
\text { imágenes } \\
\text { del cerebro } \\
\text { antes de la } \\
\text { intervención } \\
\text { y durante } \\
\text { una tarea } \\
\text { fonológica. }\end{array}$ & $\begin{array}{l}\text { La intervención de instrucción } \\
\text { impulsó enseñanza } \\
\text { fonológica de la lectura en un } \\
\text { taller de ciencias / lectura. } \\
\text { Comparó el desarrollo } \\
\text { temprano, la historia de } \\
\text { instrucción, administraciones } \\
\text { repetidas de una batería de } \\
\text { pruebas, y la respuesta a la } \\
\text { intervención de instrucción }\end{array}$ & $\begin{array}{l}\text { Mejora de las } \\
\text { habilidades fonológicas } \\
\text { (manipulación } \\
\text { fonológica, memoria y } \\
\text { decodificación } \\
\text { fonológica), en la } \\
\text { comprensión de lectura } \\
\text { en silencio, y } \\
\text { dificultades } \\
\text { considerables con las } \\
\text { habilidades de escritura } \\
\text { (escritura a mano, } \\
\text { ortografía, y la } \\
\text { velocidad de } \\
\text { componer). }\end{array}$ \\
\hline
\end{tabular}

\section{Discusión y conclusiones}

El aumento de publicaciones puede estar relacionado con la aprobación en el año 2004 de la Ley de Discapacidad (IDEA) en EEUU, que supuso invalidar el criterio de discrepancia como el único para el diagnóstico de las DEA y el impulso del modelo RTI como alternativa basada en la evidencia empírica.

Es relevante el impulso tomado por estos estudios en España a partir de la aprobación en 2006 de la LOE que reconoce el derecho de los alumnos con dificultades específicas de aprendizaje, y, en concreto, el desarrollo normativo en Canarias (Resolución de 9 de febrero de 2011, por la que se dictan instrucciones sobre los procedimientos y los plazos para la atención educativa del alumnado con necesidades específicas de apoyo educativo en los centros escolares de la Comunidad Autónoma de Canarias). Canarias es la única Comunidad Autónoma que recoge en su normativa programas preventivos de refuerzo, especificando que estarán dirigidos al alumnado de Educación Infantil y primer ciclo de Educación Primaria en riesgo de presentar dificultades para avanzar con normalidad en los aprendizajes básicos de lectura, escritura y cálculo aritmético. Estos programas consistirán en la intervención sobre este alumnado en grupos de tres a cinco escolares durante 35 minutos diarios (EURYDICE, 2012).

La revisión bibliográfica de Wanzek y Vaughn, (2007) y el metaanálisis de Suggate (2010) sugieren que las intervenciones en lectura en estudiantes con dificultades en su aprendizaje son más eficaces cuando se realizan en infantil y primer grado, y con efectos más altos cuando se realizan en grupos pequeños. Las intervenciones fonéticas son más eficaces hasta el grado uno y luego son más eficaces las intervenciones en comprensión. En otro estudio de revisión (Weiser y Mathes, 2011), se da apoyo empírico al uso de la instrucción de codificación en el aumento del rendimiento de estudiantes con dificultades lectoras y para mejorar las habilidades de lectura y escritura de los alumnos con problemas de aprendizaje. Los estudios de cohortes realizados en grupos de estudiantes con dificultades en lectura basados en el modelo RTI de respuesta del alumno a la instrucción, llevados a cabo durante $\mathrm{k}-1^{\circ}$, muestran que los resultados de lectura mejoran significativamente al final del $2^{\circ}$ grado. 
Desde un enfoque preventivo del modelo RTI, Vellutino y sus colaboradores (2008) plantean que las dificultades de lectura pueden ser detectadas tempranamente, por tanto, se puede iniciar la intervención en infantil, frente a la propuesta de intervención en cursos posteriores de un enfoque clasificatorio del modelo RTI. Esta perspectiva preventiva trata de intervenir lo antes posible para evitar las limitaciones de las habilidades de alfabetización que pudieran surgir, si desde el principio los niños y niñas reciben los apoyos complementarios que precisan, y aumentar así la eficacia de las intervenciones.

La eficacia en la detección inicial del modelo RTI se ha encontrado que es mayor que la que puede obtenerse con cualquiera de las pruebas psicométricas. En España, con el apoyo del Gobierno de las Islas Canarias, (Jiménez et al., 2010) ha implementado este modelo y prueba la eficacia del Nivel 2 de intervención utilizando una amplia muestra de alumnos de primer curso de primaria. La aplicación del plan de estudios PREDEA, en pequeños grupos a niños clasificados en riesgo de dificultades tempranas de lectura, indica mayores puntuaciones en el conocimiento del sonido de las letras, en comprensión oral, el tipo de sonido inicial y en fluidez Lectora. Otro estudio español desde un enfoque cognitivolingüístico, en la Universidad de Málaga (González, Martin, y Delgado, 2011), plantea si la intervención temprana, con la sistematización, estructuración y priorización de los contenidos instruccionales psicolingüísticos a través del currículo, mejora el rendimiento en lectura y escritura. En este estudio comprueba que una de las medidas más eficaces para mejorar el rendimiento en lectoescritura de los alumnos con riesgo de presentar dificultades de aprendizaje es a través de la instrucción sistemática de las habilidades lingüísticas y metalingüísticas desde edades tempranas. De nuevo se contempla la importancia de la intervención temprana, explícita y programada de la lectoescritura como herramienta de prevención de las dificultades de aprendizaje.

González et al. (2011) hace referencia a programas concretos como Sucess For All (SFA) y el California Early Learning Literacy (CELL), ambos se aplican desde los tres años de edad con el fin de prevenir las futuras dificultades de aprendizaje y mitigar el alto índice de fracaso escolar. A las mismas conclusiones llega (Kamps et al., 2008): los mayores rendimientos de la instrucción en lectura se dan en intervenciones más explícitas y dirigidas, y en grupos pequeños. En concreto, la investigación de estudio de caso (Berninger, 2000) analizó la intervención en lectura implementada en un taller de ciencias que impulsó la enseñanza fonológica, y se pudo constatar que procuró beneficios a los niños con dificultades en habilidades de lectura en la mejora de sus habilidades fonológicas (manipulación, memoria y decodificación fonológica) y en sus habilidades de escritura (escritura manual, ortografía y velocidad).

En resumen, la intervención eficaz en la enseñanza de la lectura parece cumplir con la detección temprana de las dificultades de lectura y en programas de intervención intensiva en pequeños grupos en los primeros años escolares basados en la instrucción del código alfabético de manera temprana, explícita y sistemática. Además, la intervención más eficaz se proporciona de principios del jardín de infantes hasta segundo grado y no después de tercer grado, y, con la suficiente intensidad, duración y apoyo permite que ningún niño se quede atrás (Foorman, Breier, y Fletcher, 2003).

Se puede concluir que es conveniente que los programas escolares incluyan tempranamente una enseñanza de la lectura sistemática basada en el método fonéticos que desarrollen la conciencia fonológica, es decir las habilidades de decodificación y codificación, el conocimiento de las letras y el establecimiento de las relaciones grafemafonema. Estas pautas de actuación basadas en la evidencia empírica a partir de estudios realizados con estudiantes en riesgo de dificultades lectoras, pueden resultar de extrema utilidad para aplicarlas en el aula ordinaria para todo el alumnado. 
Aquellos alumnos que presentan un ritmo más lento en el aprendizaje de la lectura o que cometen mayor número de errores, pueden mejorar en sus resultados de lectura con una instrucción explícita que facilite el mayor y mejor conocimiento de las reglas de conversión letra-sonido necesarios para utilizar la ruta fonológica en el procesamiento de la lectura de las palabras.

Desde el punto de vista de la prevención es imprescindible la individualización de la enseñanza, pues no es posible hablar de intervenciones generalizables para todos los estudiantes. La respuesta educativa en lectura más adecuada es aquella que mejor responde a las necesidades de cada estudiante. Se logra teniendo en cuenta una perspectiva multidimensional del proceso y de sus miembros, niños, padres, profesores y administración educativa. La participación de la familia es imprescindible en la estimulación de sus hijos y en la ayuda en los programas de apoyo. La capacitación profesional de los docentes y especialistas con programas de formación en instrucción de la lectura, en atención especializada y colaboración con las familias, que trabajan coordinados entre sí.

Es preciso que las administraciones educativas regulen un sistema educativo que contemple las medidas destinadas a ayudar a adquirir los mecanismos de base de la lectura en las etapas iniciales del aprendizaje, y en acompañar después con medidas de apoyo y compensación durante toda la vida escolar si las dificultades para leer y escribir persisten. Estas regulaciones deben contemplar la atención educativa especializada integrada en el aula ordinaria que permita a los niños con dislexia estar perfectamente integrados en los programas escolares normales y no, como ocurre con cierta frecuencia, ser víctimas de exclusión y/o de fracaso escolar.

\section{Referencias}

American Psychiatric Association (2013). Diagnostic and statistical manual of mental disorders : DSM-5 (5th ed.). Arlington: American Psychiatric Publishing.

Alegría, J. (2006) Por un enfoque psicolingüístico del aprendizaje de la lectura y sus dificultades -20 años después-. Infancia y Aprendizaje, 2006, 29 (1), 93-111

Berninger, V. W. (2000). Dyslexia the invisible, treatable disorder: The story of Einstein's ninja turtles. Learning Disability Quarterly, 23(3), 175-195. doi:10.2307/1511163

Berninger, V. W., Abbott, R. D., Vermeulen, K., Ogier, S., Brooksher, R., Zook, D.,... Lemos, Z. (2002). Comparison of faster and slower responders to early intervention in reading: Differentiating features of their language profiles. Learning Disability Quarterly, 25(1), 59-76. doi:10.2307/1511191

Caemmerer, J. M., \& Keith, T. Z. (2015). Longitudinal, reciprocal effects of social skills and achievement from kindergarten to eighth grade. Journal of School Psychology, 53(4), 265-281. doi:10.1016/j.jsp.2015.05.001

Carreker, S., \& Joshi, R. M. (2010). Response to intervention: Are the Emperor's clothes really new? Psicothema, 22(4), 943-948.

Consejería de Educación, Resolución 9 de febrero de 2011, por la que se dictan instrucciones sobre los procedimientos y los plazos para la atención educativa del alumnado con necesidades específicas de apoyo educativo en los centros escolares de la Comunidad Autónoma de Canarias. Boletín Oficial de Canarias, 24 de febrero de 2011, núm. 040.

Cuetos, F. (2008) Psicología de la lectura. Madrid: Editorial Escuela Española. 
Foorman, B. R., Breier, J. I., \& Fletcher, J. M. (2003). Interventions aimed at improving reading success: An evidence-based approach. Developmental Neuropsychology, 24(2-3), 613-639.

Gonzalez, M. J., Martin, I., \& Delgado, M. (2011). Early intervention of the reading and the writing in children with learning disabilities. Revista Latinoamericana De Psicologia, 43(1), 35-44.

Jimenez, J. E., Rodriguez, C., Crespo, P., Gonzalez, D., Artiles, C., \& Alfonso, M. (2010). Implementation of Response to Intervention (Rtl) Model in Spain: An example of a collaboration between Canarian universities and the department of education of the Canary Islands. Psicothema, 22(4), 935-942.

Jitendra, A. K., Edwards, L. L., Starosta, K., Sacks, G., Jacobson, L. A., \& Choutka, C. M. (2004). Early reading instruction for children with reading difficulties: Meeting the needs of diverse learners. Journal of Learning Disabilities, 37(5), 421-439. doi:10.1177/00222194040370050501

Kamps, D., Abbott, M., Greenwood, C., Wills, H., Veerkamp, M., \& Kaufman, J. (2008). Effects of Small-Group Reading Instruction and Curriculum Differences for Students Most at Risk in Kindergarten Two-Year Results for Secondary- and Tertiary-Level Interventions. Journal of Learning Disabilities, 41(2), 101-114. doi:10.1177/0022219407313412

Lyon, G. R. (1996). Learning disabilities. Future of Children, 6(1), 54-76. doi:10.2307/1602494

Miles, T. R. (2004). Algunos problemas en determinar la prevalencia de la dislexia. Revista Electrónica de Investigación Psicoeducativa y Psicopedagogía, 2(2), 5-12. Recuperado de http://www.investigacionpsicopedagogica.org/revista/articulos/4/espannol/Art_4_43.pdf

Ministerio de Educación (2012) La atención al alumnado con dislexia en el sistema educativo en el contexto de las necesidades específica de apoyo educativo (2012). España: MINISTERIO DE EDUCACIÓN, CULTURA Y DEPORTE. Centro Nacional de Innovación e Investigación Educativa. Eurydice España-REDIE Red Española de Información sobre Educación (CNIIE-MECD)

Ministerio de Educación. (2006) Ley Orgánica 2/2006 de 3 de mayo, de Educación. Boletín Oficial del Estado, 4 de mayo de 2006, núm. 106, pp. 17158-17207.

Ministerio de Educación. (2002) Ley Orgánica 10/2002 de 23 de diciembre, de Calidad de la Educación. Boletín Oficial del Estado, 24 de diciembre de 2002, núm. 307, pp. 4518845220.

Mioduser, D., Tur-Kaspa, H., \& Leitner, I. (2000). The learning value of computer-based instruction of early reading skills. Journal of Computer Assisted Learning, 16(1), 54-63. doi:10.1046/j.1365-2729.2000.00115.x

O'Connor, E. A., \& Simic, O. (2002). The effect of Reading Recovery (R) on special education referrals and placements. Psychology in the Schools, 39(6), 635-646. doi:10.1002/pits.10061

O'Connor, R. E., Bocian, K. M., Sanchez, V., \& Beach, K. D. (2014). Access to a Responsiveness to Intervention Model: Does Beginning Intervention in Kindergarten Matter? Journal of Learning Disabilities, 47(4), 307-328. doi:10.1177/0022219412459354 
Roberts, G. J., Solis, M., Ciullo, S., McKenna, J. W., \& Vaughn, S. (2015). Reading Interventions With Behavioral and Social Skill Outcomes: A Synthesis of Research. Behavior Modification, 39(1), 8-42. doi:10.1177/0145445514561318

Serrano, M. D. (2005). Disléxicos en español: papel de la fonología y la ortografía. Universidad de Granada.

Shaywitz, S. E., Morris, R., \& ShaywitZ, B. A. (2008). The education of dyslexic children from childhood to young adulthood Annual Review of Psychology (Vol. 59, pp. 451-475).

Soriano, M., Miranda, A., \& Cuenca, I. (1999). Intervención psicopedagógica en las Dificultades del aprendizaje escolar. REVISTA DE NEUROLOGÍA, 28 (Supl 2), S 94-100.

Suggate, S. P. (2010). Why What We Teach Depends on When: Grade and Reading Intervention Modality Moderate Effect Size. Developmental Psychology, 46(6), 15561579. doi:10.1037/a0020612

Terras, M. M., Thompson, L. C., \& Minnis, H. (2009). Dyslexia and Psycho-social Functioning: An Exploratory Study of the Role of Self-esteem and Understanding. Dyslexia, 15(4), 304-327. doi:10.1002/dys.386

Tran, L., Sanchez, T., Arellano, B., \& Swanson, H. L. (2011). A Meta-Analysis of the RTI Literature for Children at Risk for Reading Disabilities. Journal of Learning Disabilities, 44(3), 283-295. doi:10.1177/0022219410378447

Vellutino, F. R., Scanlon, D. M., Zhang, H., \& Schatschneider, C. (2008). Using response to kindergarten and first grade intervention to identify children at-risk for long-term reading difficulties. Reading and Writing, 21(4), 437-480. doi:10.1007/s11145-0079098-2

Wanzek, J., \& Vaughn, S. (2007). Research-based implications from extensive early reading interventions. School Psychology Review, 36(4), 541-561.

Weiser, B., \& Mathes, P. (2011). Using Encoding Instruction to Improve the Reading and Spelling Performances of Elementary Students At Risk for Literacy Difficulties: A Best-Evidence Synthesis. Review of Educational Research, 81(2), 170-200. doi:10.3102/0034654310396719

Yu, J. W., Buka, S. L., McCormick, M. C., Fitzmaurice, G. M., \& Indurkhya, A. (2006). Behavioral problems and the effects of early intervention on eight-year-old children with learning disabilities. Maternal and Child Health Journal, 10(4), 329-338. doi:10.1007/s10995-005-0066-7 